\title{
Alimentação como tratamento coadjuvante para pessoas com fibromialgia: uma revisão integrativa
}

Feeding as a supporting treatment for people with fibromyalgia: an integrative review

\section{Alimentación como tratamiento secundario para personas con fibromialgia: una revisión integradora}

Danielle Mendonça Jatobá ${ }^{1 *}$, Luiza Jatobá de Hollanda ${ }^{2}$, Ana Clara Veloso de Assis ${ }^{1}$, Brenda Tayrine Tavares Souza1, Bruna Santana Tavares ${ }^{1}$, Jackson Gois Teixeira ${ }^{1}$, Jhully Leandro Lirman ${ }^{1}$, Mariana Cândida Silveira1, Thaynara Ferreira Alves ${ }^{1}$, Albênica Paulino dos Santos Bontempo.

\section{RESUMO}

Objetivo: Observar os benefícios da alimentação saudável no tratamento coadjuvante da fibromialgia. Métodos: Trata-se de uma revisão integrativa da literatura realizada na Biblioteca Virtual em Saúde utilizando artigos publicados nos últimos 10 anos e disponíveis na íntegra. A seleção procedeu 3 etapas: leitura do título, resumo e integral. Resultados: Inicialmente foram encontrados 63 artigos, destes foram selecionados 23 pelos critérios de elegibilidade. Observou-se melhora significativa dos sintomas nos estudos em que pacientes aderiram a uma dieta sem glúten ou que incluíram na alimentação alimentos fonte de magnésio, cálcio e triptofano. Dietas vegetarianas ou veganas e hipocalóricas também mostraram benefícios no controle e redução dos sintomas, assim como as dietas anti-inflamatórias, que são caracterizadas pelo alto consumo de frutas e vegetais, ingestão moderada de proteína e consumo restrito de pães e grãos. Já as dietas próinflamatórias, ricas em gorduras, açúcares e produtos processados, contribuíram para uma piora dos sintomas da fibromialgia. Considerações finais: Alimentos e dietas específicas podem ser utilizados para amenizar ou até mesmo controlar sintomas da fibromialgia. Pacientes que conseguiram modificar seu estilo de vida e seguir uma alimentação saudável tiveram melhores resultados no tratamento.

Palavras-chave: Fibromialgia, Terapêutica, Dieta.

\begin{abstract}
Objective: To observe the benefits of healthy feeding in the supporting treatment of fibromyalgia. Methods: An integrative review of the literature carried out in the Virtual Health Library with articles published in the last 10 years, available in full. The selection proceeded in 3 stages, reading of the title, abstract and full article. Results: Initially, 63 articles were found, of which 23 were selected by eligibility criteria. There was a significant improvement in symptoms in studies in which patients adhered to a gluten-free diet or included foods that were a source of magnesium, calcium, and tryptophan. Vegetarian or vegan and hypocaloric diets also showed benefits in controlling and reducing symptoms, as well as anti-inflammatory diets that are characterized by high fruit and vegetable intake, moderate protein intake, and restricted consumption of breads and grains. On the other hand, pro-inflammatory diets, rich in fats, sugars, and processed products, contributed to a worsening of the symptoms of fibromyalgia. Final considerations: Specific foods and diets can be used to soften or even control symptoms of fibromyalgia. Patients who have managed to modify their lifestyle and follow a healthy diet have better results in treatment.
\end{abstract}

Key words: Fibromyalgia, Therapeutics, Diet.

${ }^{1}$ Centro Universitário do Distrito Federal (UDF), Brasília - DF.

*E-mail: danijatoba.nutricao@gmail.com

2 Universidade de Brasília (UnB), Brasília - DF.

SUBMETIDO EM: 1/2022

ACEITO EM: 1/2022

PUBLICADO EM: 1/2022 


\section{RESUMEN}

Objetivo: Observar los beneficios de la alimentación en el tratamiento de la fibromialgia. Métodos: Revisión integradora realizada en la Biblioteca Virtual en Salud con artículos publicados en los últimos 10 años y disponible en su totalidad. La selección se desarrolló en 3 etapas, lectura del título, resumen y artículo completo. Resultados: Inicialmente se encontraron 63 artículos, de los cuales 23 fueron seleccionados por criterios de elegibilidad. Hubo una mejora significativa en los síntomas en estudios que los pacientes siguieron una dieta sin gluten o incluyeron alimentos que eran una fuente de magnesio, calcio y triptófano. Las dietas vegetarianas o veganas y hipocalórico también mostraron beneficios para controlar y reducir los síntomas, así como las dietas antiinflamatorias, se caracterizan por una alta ing esta de frutas y verduras, ingesta moderada de proteínas y un consumo restringido de panes y cereales. Por otro lado, las dietas proinflamatorias, ricas en grasas, azúcares y productos procesados, contribuyeron a un agravamiento de los síntomas de la fibromialgia. Consideraciones finales: Se pueden usar alimentos y dietas específicas para suavizar y controlar los síntomas de la fibromialgia. Los pacientes que han logrado modificar su estilo de vida y una dieta saludable tienen mejores resultados en el tratamiento.

Palabras clave: Fibromialgia, Terapéutica, Dieta.

\section{INTRODUÇÃO}

A Fibromialgia (FM) é uma síndrome reumática crônica predominante em mulheres e caracterizada por dor musculoesquelética generalizada e difusa. Pessoas com FM apresentam sintomas como distúrbios do sono, fadiga, Síndrome do Intestino Irritável (SII), transtornos do humor, cefaleia, entre outros (ANDRETTA A, et al., 2019; COSKUN B, 2019). Sendo inúmeros os sintomas relatados por pessoas com FM, esta síndrome já foi denominada como "síndrome de tudo irritável" (BARKER K, 2005).

A síndrome da FM não é diagnosticada por exames laboratoriais nem de imagem, mas sim pelo exame clínico, onde o médico utiliza o exame físico e a anamnese detalhada, o que gera dúvidas e incertezas nos pacientes sobre o diagnóstico (BOULTON T, 2019; OLIVEIRA JO e RAMOS JV, 2019). Seu tratamento é multidisciplinar e inclui terapia farmacológica e não farmacológica visando o alívio da dor crônica. A Qualidade de Vida (QV) de pessoas com FM é extremamente af etada devido a sintomas como a ansiedade, alterações de humor, obesidade e síndrome metabólica (OLIVEIRA JO e RAMOS JV, 2019; COSKUN B, 2019).

A síndrome da FM atinge cerca de 2,5\% da população brasileira. Estudos mostram que nos Estados Unidos a prevalência chega a $5 \%$ das mulheres e $4,7 \%$ da população em alguns países da Europa (SOUZA JB, et al., 2017). Os pacientes com FM são identificados através dos pontos dolorosos espalhados pelo corpo (acima de 11) com sensibilidade de $84 \%$, pontos difusos dolorosos e especificidade de $87 \%$, pontos que mostram maior assertividade, o que confere certeza diagnóstica de $84 \%$ quando positivo (HEYMANN RE, et al., 2017).

Uma boa alimentação representa um papel fundamental no controle dos sintomas das doenças crônicas, pois, junto com a nutrição, é requisito básico para que se possa promover e proteger a saúde da população. (BATISTA ED, et al., 2016; JAIME PC, et al., 2011). Para pessoas com FM, a nutrição desempenha um fator de extrema importância, uma vez que a dieta pode influenciar nos sintomas da doença. $A$ análise do comportamento alimentar ajuda a entender os benefícios de alguns alimentos no controle e na melhora dos sintomas e, consequentemente, na QV (RODRíGUEZ MD, et al., 2017).

Cuidar da alimentação de pessoas com FM pode ajudar a controlar os sintomas dessa síndrome, pois sabemos, por exemplo, que a caf eína interfere na qualidade do sono. Distúrbios do sono são um dos sintomas da FM e podem ser controlados ou amenizados com a alimentação, incluindo-se ou excluindo-se alguns alimentos da dieta, assim como o sobrepeso, que também af eta pessoas com FM e pode ser controlado com uma alimentação saudável e balanceada (SIENA LR e MARRONE L, 2018).

Diante do exposto, este estudo teve por objetivo observar os benefícios de uma alimentação saudável como tratamento coadjuvante para pessoas com FM através de uma revisão integrativa. 


\section{MÉTODOS}

Trata-se de um estudo de revisão integrativa. No primeiro momento, foi realizado um levantamento bibliográfico nas principais bases de dados da Biblioteca Virtual em Saúde por conter as principais revistas científicas indexadas e foram utilizadas a Literatura Latino-Americana e do Caribe em Ciências da Saúde (LILACS), a Scientific Electronic Library Online (SciELO) e a Público/editora Medline (PubMed). Foram seguidas as orientações do Relatório Preferidos para Revisões Sistemáticas e Meta-Análises (PRISMA) e foram utilizados os descritores "fibromialgia", "terapêutica", e "dieta" e os filtros: texto disponível na íntegra, assunto principal "fibromialgia", publicado nos últimos 10 anos nos idiomas inglês, português e espanhol. Devido a pouca literatura sobre o tema, foi necessário expander o período da pesquisa de 5 para 10 anos.

Na pesquisa foram incluídos artigos de revisão sistemática, revisão bibliográfica, estudo de caso e ensaio clínico, e excluídos comentários aos autores, cartas editoriais, relatos de caso e artigos que não estavam disponíveis na íntegra. As buscas foram realizadas por quatro pesquisadores e a seleção dos estudos teve três etapas: leitura dos títulos, leitura dos resumos e leitura na íntegra. Por fim, foram extraídos os resultados e conclusões mais relevantes para a elaboração do artigo de revisão.

Como benefícios, trazemos uma síntese das informações sobre o tratamento dos sinais e sintomas da FM através da alimentação, proporcionando conhecimento sobre alimentos que melhoram os sintomas e, consequentemente, a QV das pessoas que sofrem com FM. Através deste estudo, poderão ser realizadas políticas públicas de alimentação e nutrição no combate aos sinais e sintomas da FM.

\section{RESULTADOS}

Foram localizados 63 artigos, em seguida, removidos os duplicados e indisponíveis. Restaram 23 após o uso dos filtros e aplicação dos critérios de elegibilidade. Os detalhes da busca estão esquematizados no fluxograma da Figura 1.

Figura 1 - Diagrama de Fluxo PRISMA.

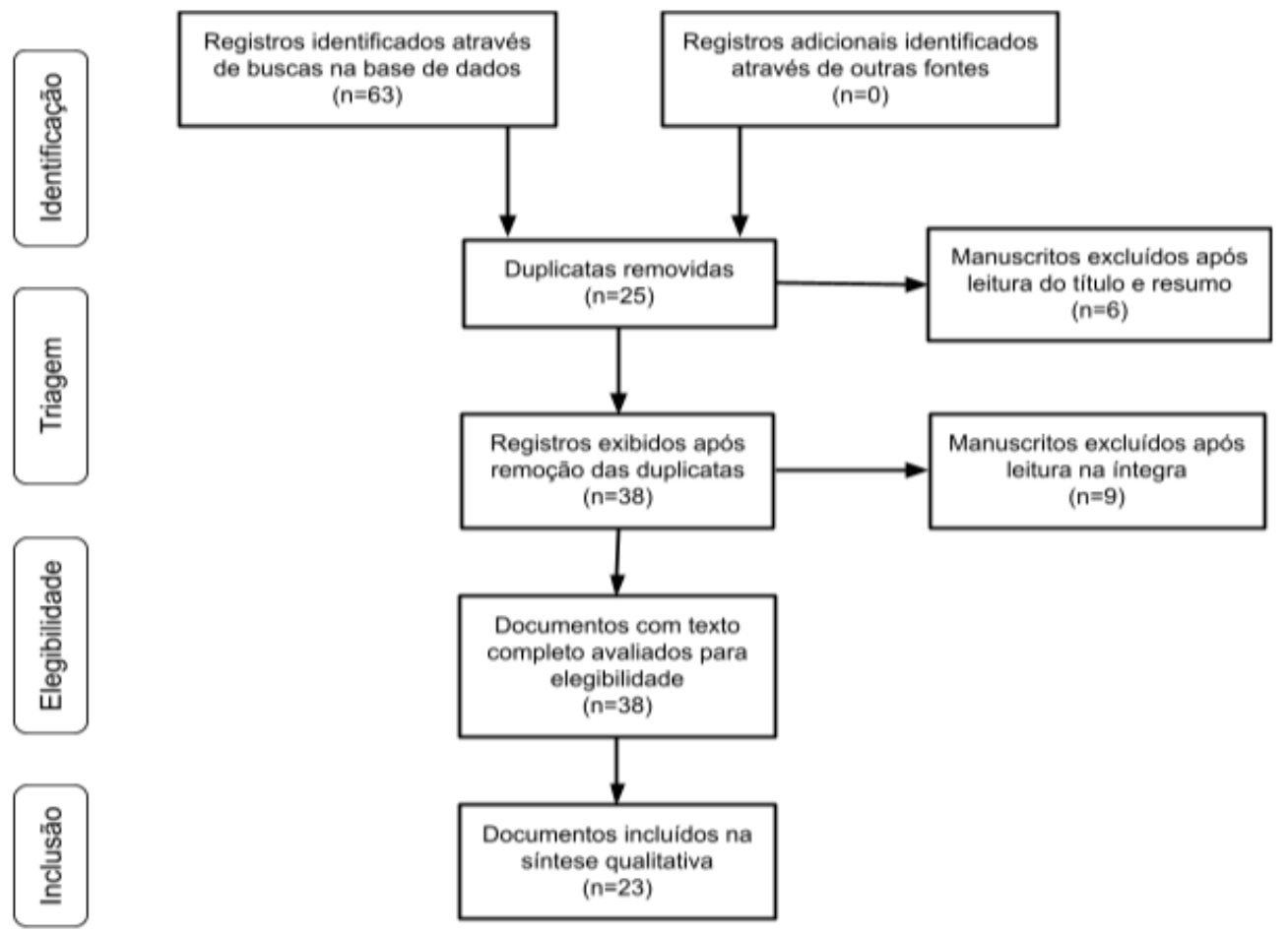

Fonte: Jatobá DM, et al., 2021.

O Quadro 1 traz uma síntese dos principais achados nos estudos selecionados para este artigo, destacando trechos relacionados à alimentação como tratamento coadjuvante nos sintomas da fibromialgia. 
Quadro 1 - Síntese dos principais achados referentes aos benefícios da alimentação saudável como tratamento coadjuvante para pessoas com FM, 2021.

\begin{tabular}{|c|c|c|}
\hline $\mathbf{N}$ & Autores (Ano) & Principais achados \\
\hline 1 & NADAL-NICOLÁS Y, et al. (2021). & $\begin{array}{l}\text { Revisão sistemática. Revisar as evidências sobre o efeito da dieta vegetariana ou vegana em pacientes com FM; } \\
\text { dieta vegetariana ou vegana parece reduzir os sintomas e melhorar a QV dos pacientes. }\end{array}$ \\
\hline 2 & PAZZI F, et al. (2021). & $\begin{array}{l}\text { Ensaio piloto randomizado duplo-cego. Avaliar os efeitos de GL e CS em parâmetros sanguíneos e medidas } \\
\text { antropométricas de mulheres com FM; não encontraram resultados que mostrassem ef eito significativo sobre os } \\
\text { parâmetros sanguíneos e antropométricos de mulheres com FM. }\end{array}$ \\
\hline 3 & PAGLIAI G, et al. (2020). & $\begin{array}{l}\text { Revisão da literatura. Resumir a relação entre FM e nutrição, explorando as evidências sobre o efeito de } \\
\text { suplementos dietéticos e intervenções; os aspectos dietéticos parecem ser uma abordagem complementar } \\
\text { promissora para o tratamento da FM. }\end{array}$ \\
\hline 4 & PAGLIAI G, et al. (2020). & $\begin{array}{l}\text { Estudo cruzado randomizado duplo-cego. Investigar os ef eitos da dieta com trigo khorasan em comparação com } \\
\text { dieta semelhante com trigo moderno semi-integral em pacientes com FM; intervenção dietética com trigo Khorasan } \\
\text { parece beneficiar os pacientes, especialmente aqueles com maior gravidade dos sintomas. }\end{array}$ \\
\hline 5 & $\begin{array}{l}\text { CORREA-RODRÍGUEZ M, et al. } \\
\qquad(2020) .\end{array}$ & $\begin{array}{l}\text { Estudo transversal. Análise de dieta anti-inflamatória e os limiares de dor e outros sintomas relacionados à FM; o } \\
\text { potencial inf lamatório da dieta pode desempenhar um papel relevante na hipersensibilidade à dor. }\end{array}$ \\
\hline 6 & LOWRY E, et al. (2020). & $\begin{array}{l}\text { Revisão sistemática. Observar as intervenções nutricionais específicas para o manejo da FM; essas intervenções } \\
\text { se mostrando mais promissoras em ensaios clínicos bem elaborados podem fornecer um protocolo de tratamento } \\
\text { ativo. }\end{array}$ \\
\hline 7 & $\begin{array}{l}\text { MARTíNEZ-RODRÍGUEZ A, et al. } \\
\text { (2020). }\end{array}$ & $\begin{array}{l}\text { Ensaio clínico randomizado e controlado. Determinar os ef eitos da dieta mediterrânea enriquecida com triptofano } \\
\text { e magnésio em variáveis psicológicas e qualidade do sono em mulheres com FM; dieta mediterrânea enriquecida } \\
\text { com triptofano e magnésio reduziu os sintomas de ansiedade, distúrbios do humor, distúrbios alimentares e } \\
\text { insatisfação com a imagem corporal, mas não melhorou a qualidade do sono. }\end{array}$ \\
\hline 8 & ANDRETTA A, et al. (2019). & $\begin{array}{l}\text { Pesquisa transversal. Determinar a ingestão alimentar e níveis séricos de magnésio e cálcio e correlacionar esses } \\
\text { minerais com dor, qualidade de vida e risco de depressão em mulheres com e sem FM.; menor ingestão dietética } \\
\text { de magnésio e cálcio se correlacionou com piora dos parâmetros de dor na FM. }\end{array}$ \\
\hline
\end{tabular}




\begin{tabular}{|c|c|c|}
\hline $\mathbf{N}$ & Autores (Ano) & Principais achados \\
\hline 9 & SILVA AR, et al. (2019). & $\begin{array}{l}\text { Revisão sistemática. Sintetizar as informações sobre o ef eito das intervenções dietéticas a partir de resultados } \\
\text { relatados pelos pacientes e inflamação em pacientes com FM; A dor em pacientes com FM parece melhorar com } \\
\text { uma dieta hipocalórica, uma dieta vegetariana crua ou uma dieta baixa em FODMAP. }\end{array}$ \\
\hline 10 & $\begin{array}{l}\text { MARTíNEZ-RODRÍGUEZ A, et al. } \\
\text { (2018). }\end{array}$ & $\begin{array}{l}\text { Estudo de desenho quase experimental. Conhecer a eficácia do tratamento fisioterapêutico associado a uma } \\
\text { intervenção dietético-nutricional lactovegetariana na lombalgia e na composição corporal de mulheres com FM; } \\
\text { Um programa de intervenção combinando exercícios de estabilização corporal e dieta lactovegetariana em } \\
\text { pacientes com FM e com dor lombar contribui para a redução da dor e melhora da composição corporal. }\end{array}$ \\
\hline 11 & $\begin{array}{l}\text { LÓPEZ-RODRÍGUEZ MM, et al. } \\
\qquad(2017) \text {. }\end{array}$ & $\begin{array}{l}\text { Estudo descritivo. Descrever os hábitos e comportamentos alimentares em pacientes com fibromialgia e comparar } \\
\text { com os saudáveis; os hábitos alimentares de pacientes com FM diferem dos saudáveis no menor consumo de } \\
\text { cereais, frutas, açúcares, refrigerantes e álcool, além de geralmente evitar mais laticínios, frutas cítricas, cereais, } \\
\text { álcool e refrigerantes. }\end{array}$ \\
\hline 12 & MARUM AP, et al. (2017). & $\begin{array}{l}\text { Ensaio clínico. Estudar os ef eitos de dietas com FODMAP sobre os sintomas da FM e peso corporal; dietas com } \\
\text { baixa fermentação de FODMAP contribui para a perda de peso e reduz a gravidade dos sintomas da FM. }\end{array}$ \\
\hline 13 & RUIZ-CABELLO P, et al. (2017). & $\begin{array}{l}\text { Estudo transversal. Avaliar os hábitos alimentares em mulheres com FM e explorar sua associação com saúde } \\
\text { mental, depressão e otimismo; ingestão diária de frutas e vegetais e ingestão moderada de peixes podem estar } \\
\text { associados a resultados psicossociais mais favoráveis em mulheres com FM, e ingestão excessiva de carnes } \\
\text { curadas e bebidas adoçadas foi relacionada a piores pontuações nos resultados de otimismo e depressão. }\end{array}$ \\
\hline 14 & SLIM M, et al. (2017). & $\begin{array}{l}\text { Ensaio clínico piloto, aberto e randomizado. Avaliar os ef eitos de uma dieta sem glúten em comparação com uma } \\
\text { dieta hipocalórica em pacientes com FM; ambas as intervenções dietéticas foram associadas a resultados } \\
\text { benéficos semelhantes na redução dos sintomas de sensibilidade ao glúten e outros resultados secundários. }\end{array}$ \\
\hline 15 & BATISTA ED, et al. (2016). & $\begin{array}{l}\text { Estudo analítico, transversal e observacional. Comparar a ingestão alimentar de mulheres com e sem FM e } \\
\text { verificar se interfere na sensação de dor e QV; Apenas a vitamina E se correlacionou com QV e percentual de } \\
\text { proteína na dieta com sensação de dor. }\end{array}$ \\
\hline 16 & MARUM AP, et al. (2016). & $\begin{array}{l}\text { Estudo longitudinal. Examinar os efeitos das dietas com baixo FODMAP nos sintomas da FM; dietoterapia } \\
\text { envolvendo restrições de FODMAP resultou em redução significativa nos distúrbios gastrointestinais e sintomas } \\
\text { da FM. }\end{array}$ \\
\hline
\end{tabular}




\begin{tabular}{|c|c|c|}
\hline $\mathbf{N}$ & Autores (Ano) & Principais achados \\
\hline 17 & CRAFT JM, et al. (2015). & $\begin{array}{l}\text { Análise qualitativa. Identificar barreiras, necessidades e preferências de intervenção de controle de peso para } \\
\text { mulheres com FM; mulheres com FM identificam barreiras para o controle do peso, incluindo as relações entre os } \\
\text { sintomas e os comportamentos de saúde, como dieta e exercícios. }\end{array}$ \\
\hline 18 & SLIM M, et al. (2015). & $\begin{array}{l}\text { Ensaio clínico randomizado. Avaliar o benefício de evitar o glúten em pacientes com FM e sensibilidade ao glúten; } \\
\text { mudanças positivas no número de sintomas de sensibilidade ao glúten. }\end{array}$ \\
\hline 19 & ISASI C, et al. (2014). & $\begin{array}{l}\text { Ensaio aberto. Descrever pacientes selecionados com FM sem DC que melhoraram quando colocados em uma } \\
\text { dieta sem glúten; melhora clínica pode ser alcançada com uma dieta sem glúten em pacientes com FM, mes mo } \\
\text { que a DC tenha sido descartada. }\end{array}$ \\
\hline 20 & CORDERO MD, et al. (2014). & $\begin{array}{l}\text { Estudo transversal. Analisar a associação entre IMC, perfil lipídico e sintomas clínicos em pacientes com FM; } \\
\text { sobrepeso e perfil lipídico podem estar associados aos sintomas da FM. }\end{array}$ \\
\hline 21 & VOLTA U (2014). & $\begin{array}{l}\text { Artigo de opinião. Avaliar o ef eito de uma dieta sem glúten em pacientes com SII e FM apresentando enterite } \\
\text { linfocítica; a retirada do glúten produziu uma melhora significativa dos sintomas funcionais, sug erindo que o glúten } \\
\text { pode ser parcialmente responsável por esse quadro clínico. }\end{array}$ \\
\hline 22 & RODRIGO L, et al. (2013). & $\begin{array}{l}\text { Estudo longitudinal prospectivo. Avaliar a eficácia de uma dieta sem glúten em mulheres com DC, SII e FM; a } \\
\text { adesão a uma dieta sem gluten pode melhorar simultaneamente os sintomas de DC e SII/FM. }\end{array}$ \\
\hline 23 & SENNA MK, et al. (2012). & $\begin{array}{l}\text { Ensaio controlado randomizado cego de desenho paralelo. Examinar se a redução de peso pode resultar na } \\
\text { melhoria do questionário de impacto da FM; perda de peso no paciente obeso com FM leva a uma melhora } \\
\text { significativa na QV. }\end{array}$ \\
\hline
\end{tabular}

Legenda: QV: qualidade de vida; FM: fibromialgia; DC: doença celíaca; SIl: síndrome do intestino irritável; SN: suplementos nutricionais ; IMC: índice de massa corporal; CT: controle; GL: Ganoderma lucidum; CS: Ceratonia siliqua; FODMAP: oligossacarídeos, dissacarídeos, monossacarídeos e polióis fermentáveis.

Fonte: Jatobá DM, et al., 2021. 


\section{DISCUSSÃO}

Como apontam Bottega FH e Fontana RT (2010), a dor está correlacionada com sof rimento humano, incapacidade e QV, o que a torna um problema de saúde pública, no qual $80 \%$ da procura pelos serviços de saúde são motivados por ela. De acordo com Siracusa R, et al. (2021), a FM é uma síndrome reumática crônica caracterizada pela dor musculoesquelética generalizada e difusa, logo, é necessário buscar mecanismos para o tratamento ou alívio dessa dor no acompanhamento multiprofissional de pacientes por ela acometidos.

Pessoas com FM sofrem com incapacidade até mesmo para atividades do dia a dia em decorrência dos sintomas da doença, dificuldade de realizar tarefas simples, como varrer a casa ou trocar de roupa. Essas incapacidades podem ser extremamente prejudiciais, não apenas fisicamente, mas interferem também no psicológico, levando a outros sinais e sintomas da FM, como ansiedade e depressão. São vários os sintomas físicos e psicológicos dessa doença crônica que interferem diretamente na QV desses indivíduos (SIRACUSA $R$, et al., 2021).

Segundo Nadal-Nicolás Y, et al. (2021), uma alimentação saudável é primordial para a promoção da QV dos pacientes portadores de doenças crônicas e que os alimentos que contém glutamato intensificam os sintomas da síndrome da FM. Com isso, os autores dizem que uma dieta vegetariana ou vegana, além de ser saudável, é rica em antioxidantes que propiciam o alívio dos sintomas da síndrome da FM e relatam que essas dietas possuem baixíssimo teor de colesterol, gorduras saturadas, apresentam um bom controle da glicose sérica. É característico dessas dietas o fornecimento de Potássio $(\mathrm{K})$, Magnésio $(\mathrm{Mg})$, fibras, boro, carotenóides, fitoquímicos e antioxidantes como as vitaminas $\mathrm{C} \mathrm{e} \mathrm{E}$, e os pacientes que aderem ao vegetarianismo, além de obterem uma nutrição adequada, ainda adquirem benefícios cardiovasculares e funcionais.

Para Correa-Rodríguez M, et al. (2020), existem tipos de dietas que podem agravar a sensibilidade à dor, corroborando com a ideia de Nadal-Nicolás Y, et al. (2021), de que a alimentação está ligada à qualidade de vida das pessoas com síndrome da FM. Para tanto, Correa-Rodríguez M, et al. (2020), classificaram dois estilos divergentes de dieta, sendo uma delas a dieta anti-inflamatória, que prioriza alimentos que combatem de forma natural os processos inflamatórios no organismo, como as frutas vermelhas, o azeite de oliva extravirgem, as castanhas e as nozes, e que pode diminuir os níveis de citocinas pró-inflamatórias, que atuam no organismo promovendo o processo inflamatório, reduzindo a hipersensibilidade à dor.

Outra dieta citada por Correa-Rodríguez M, et al. (2020), é a pró-inflamatória, cujas citocinas presentes nos alimentos desencadeiam processos clínicos característicos da doença, tais como a dor, perda de sono, ansiedade e fadiga. Os autores destacam também um ponto sobre a dieta vegana rica em lactobactérias que contribui para redução da dor e melhora a qualidade do sono nos portadores de fibromialgia.

Cabe ressaltar que, de acordo com Pagliai G, et al. (2020) e Pazzi F, et al. (2021), a suplementação alimentar de vitamina $D$ mostrou resultados favoráveis na redução dos sintomas da FM como dor crônica, ansiedade, depressão, função cognitiva, padrão de sono e sintomas gastrointestinais, e deve ser levada em consideração no manuseamento da doença já que $40 \%$ das pessoas com fibromialgia investigadas apresentam-se deficientes da vitamina, destacando a importância da dieta suplementar de vitaminas, Mg, ferro e probióticos, que são bactérias vivas que auxiliam no equilíbrio da microbiota intestinal e no fortalecimento do sistema imunológico e atuam reduzindo sintomas gastrointestinais como náusea e inchaço, diminuindo, assim, o nível de dor.

Para Lowry E, et al. (2020), as intervenções dietéticas precisam ser estudadas a fundo, já que apresentam aspectos positivos como, por exemplo, o vegetarianismo, que melhora a inflamação, e o estresse oxidativo, que diminui a produção de radicais livres que podem atuar de forma desequilibrada no organismo, e a suplementação de vitamina $D$ que apresenta uma melhora considerável na função física, pois é importante para imunidade, além de regular a catelicidina, um antibiótico natural de amplo espectro, enfatizando-se assim, a importância de aprofundar os estudos sobre a dieta suplementar já que Pagliai G, et al. (2020), também fala sobre essa vitamina. 
A baixa ingestão alimentar e, consequentemente, a deficiência de vitaminas e minerais na dieta, especialmente $\mathrm{Mg}$, que desempenha um papel importante na FM uma vez que várias manifestações dessa condição como fadiga, fraqueza muscular, SIl e parestesia, são semelhantes aos sintomas da deficiência desse mineral, e Cálcio $(\mathrm{Ca})$, está relacionada com a piora da dor crônica e da FM, segundo Andretta A, et al. (2019). No estudo de Ruiz-Cabello P, et al. (2017), uma ingestão frequente de frutas e vegetais e moderada de peixe pode estar associada a resultados favoráveis em mulheres com FM no que diz respeito à saúde mental. Como cita Correa-Rodríguez M, et al. (2020), o ômega 3 presente no peixe atua no combate dos processos inflamatórios do organismo de forma natural, assim como na dieta anti-inflamatória. Dessa forma, os autores conseguiram avaliar a associação de sintomas como dor, saúde mental, depressão e otimismo em mulheres com FM relacionados com maior ou menor ingestão de alimentos específicos.

Estudar a ingestão alimentar de mulheres com e sem fibromialgia para analisar a sensação de dor e QV foi o objetivo do artigo escrito por Batista ED, et al. (2016), onde, para avaliação, foram analisadas as calorias totais, carboidratos, proteínas, lipídeos, vitaminas (A, C, B12, D e E) e minerais (folato, selênio, zinco, ferro, $\mathrm{Ca}$ e $\mathrm{Mg}$ ). Apenas a vitamina $\mathrm{E}$ teve resultado se correlacionando com a $\mathrm{QV}$, e o percentual de proteína na dieta se correlacionou com a sensação de dor. Os resultados do estudo de Batista ED, et al. (2016), mostram a importância de uma alimentação adequada e saudável para melhorar os sintomas da FM e que manter uma dieta saudável e balanceada com vitaminas e minerais é importante para diminuir os sintomas da doença.

Silva AR, et al. (2019), possuem ideias que condizem e se relacionam com as de Marum AP, et al. (2017) e Pazzi F, et al. (2021), que afirmam que a dieta com baixo FODMAP (oligossacarídeos, dissacarídeos, monossacarídeos e polióis fermentáveis), além de melhorar a dor, ainda atua positivamente na QV dos portadores de FM, promovendo a diminuição dos sintomas de ansiedade e depressão, fadiga e melhora do sono. Essa dieta, além de reduzir índices de dor, também promove a perda de peso e contribui significativamente na diminuição dos sintomas da FM, resultando em uma terapia nutricionalmente balanceada com benefícios sintomáticos somáticos e viscerais e exerce papel de destaque em relação aos distúrbios gastrointestinais e SII.

Um ensaio de intervenção dietética foi realizado por Pagliai G, et al. (2020), para avaliar os ef eitos de uma dieta com trigo Khorasan em comparação a uma dieta com trigo moderno semi-integral orgânico. $O$ estudo apontou resultados satisfatórios e mostrou que o trigo Khorasan é benéfico aos pacientes com maior gravidade dos sintomas da FM pois leva a uma melhora da dor, dos sintomas gastrointestinais, da qualidade do sono e do perfil inflamatório dos pacientes. Já Pazzi F, et al. (2021), com o objetivo de observar os efeitos da Ganoderm Lucidum (GL), cogumelo utilizado na medicina chinesa, e Ceratonia Siliqua (CS), cuja farinha é rica em fibras e uma fonte rica em carboidratos, proteínas e minerais, em mulheres com FM, avaliaram alguns parâmetros e puderam concluir que GL e CS não apontaram utilidade como Suplementos nutricionais (SN) para a melhora dos parâmetros sanguíneos e medidas antropométricas nessas pacientes.

Recentemente, uma nova síndrome foi introduzida no espectro das doenças relacionadas ao glúten e denominada sensibilidade ao glúten não celíaco. Volta U (2014), relata que foram avaliados os ef eitos de uma dieta sem glúten na evolução clínica de pacientes com SII associada a FM e observaram que a retirada do glúten trouxe melhora leve, mas significativa dos sintomas funcionais, o que sugere ser o glúten responsável, em parte, pelo quadro clínico desses pacientes. Esse estudo foi realizado em pacientes com enterite linfocítica, o que indica que essa condição pode ser útil para identificar os pacientes que realmente se beneficiam com a dieta sem glúten. Portanto, para pacientes com SIl associada à FM e mucosa intestinal sem alterações, segundo Volta U (2014), a dieta sem glúten não é apropriada.

Segundo o estudo de Slim M, et al. (2015), distúrbios gastrointestinais são frequentes em pacientes com FM e normalmente atribuídos à SIl, mas também podem ser semelhantes aos sintomas dos distúrbios relacionados ao glúten, como a Doença celíaca (DC). A dieta sem glúten está relacionada à limitação do consumo de alguns alimentos, reduzindo a ingestão de carboidratos, fibras e alguns minerais. Assim, Rodrigo $\mathrm{L}$, et al. (2013), observaram que uma dieta sem glúten para mulheres com DC categorizadas como pacientes com SIl e síndrome da FM pode melhorar simultaneamente os sintomas, tanto da DC como da SII e da síndrome da FM. Contudo, Isasi C, et al. (2014), fizeram um estudo em pacientes com FM sem DC que 
apresentaram melhora quando submetidos a uma dieta sem glúten, em que foi possível observar que a sensibilidade ao glúten pode ser uma forma tratável da FM.

Os estudos de Volta U (2014), Isasi C, et al. (2014), Rodrigo L, et al. (2013) e Slim M, et al. (2015), que falam sobre a retirada do glúten da dieta dos pacientes com FM, sempre trazem pessoas com uma outra patologia associada à FM, como, por exemplo, a enterite linfocítica citada no artigo do Volta U (2014). Dessa forma, conseguimos perceber que uma dieta sem glúten é benéfica para a patologia associada à FM e, consequentemente, tratando essa patologia, consegue-se reduzir ou amenizar os sintomas da FM.

Comparando uma dieta sem glúten e uma dieta hipocalórica, no estudo de Slim M, et al. (2017), ambas apresentaram bons resultados no que diz respeito à redução de sintomas, principalmente nos sintomas de sensibilidade ao glúten. Em contrapartida, o estudo de Craft JM, et al. (2015), demonstrou que uma dieta restrita em energia para pacientes obesos com FM resultou na melhora de sintomas da doença como depressão, qualidade do sono e dor. Apesar disso, ambas as intervenções dietéticas mostraram benefícios na redução dos padrões de intensidade da dor, o que sugere uma correlação entre obesidade e dor crônica.

Cordero MD, et al. (2014), avaliaram a relação entre Índice de massa corporal (IMC) e perfil lipídico associados aos sintomas clínicos da fibromialgia e identificaram uma correlação significativa entre maior IMC e percepção de dor. Segundo o autor, a alimentação inadequada, a inatividade física e o excesso de peso e de adiposidade são responsáveis pelo desenvolvimento de dislipidemias, o que por si só é um fator prejud icial aos sintomas de dor da fibromialgia (CORDERO MD, et al., 2014).

O estudo também destacou que o colesterol acima dos padrões de referência está associado a duração da dor, pontos sensíveis, pontuação total no Questionário de impacto da FM (FIQ), ansiedade e depressão, enquanto que o aumento dos triglicerídeos está associado a Escala Visual Analógica (VAS), pontuação total do FIQ, dor e fadiga. Esses resultados são importantes para determinar que pacientes portadores da síndrome da FM devem modificar o estilo de vida com uma dieta adequada e um programa de exercícios físicos com estratégias de perda de peso (CORDERO MD, et al., 2014). O IMC, a flutuação de peso e o consumo de produtos fitoterápicos foram mais altos no estudo de López-Rodríguez MM, et al. (2017), mostrando uma correlação importante com os estudos de Cordero MD, et al. (2014) e Craft JM, et al. (2015), que também citam o IMC e a obesidade em relação à FM.

Senna MK, et al. (2012), observaram que pacientes que perderam peso e reduziram Interleucina-6 (IL 6) e Proteína C Reativa (PCR) melhoraram a qualidade do sono, QV, depressão, diminuição do escore FIQ e contagem de pontos sensíveis de dor quando comparados ao grupo de controle do estudo. Entretanto, ambos os grupos receberam alimentação adequada e o resultado satisfatório obtido se deve à restrição calórica e não aos alimentos e padrões alimentares. O estudo de Senna MK, et al. (2012), sugere ainda que a redução de peso é fundamental para o tratamento da fibromialgia, uma vez que a obesidade aumenta significativamente a carga de incapacidade da doença. A melhora da sensibilidade à dor evidenciada nas áreas inferiores do corpo é influenciada pela carga mecânica de carregar peso extra, corroborando com os resultados dos estudos de Cordero MD, et al. (2014), López-Rodríguez MM, et al. (2017) e Craft JM, et al. (2015), no qual mencionam a obesidade relacionada à sensibilidade à dor.

No estudo de Martínez-Rodríguez A, et al. (2018), foi observada uma relação entre a massa muscular e a diminuição da dor em mulheres com lombalgia e FM. Uma dieta lactovegetariana isocalórica associada a exercícios físicos mostrou melhoras significativas na composição corporal e na redução da dor, o que corrobora com o estudo de Senna MK, et al. (2012), que concluiu que a QV dos pacientes com FM melhorou significativamente após a redução do peso corporal. Outros sintomas da FM também podem ser amenizados com a redução do peso como, por exemplo, a depressão e a qualidade do sono, sugerindo, assim, que o tratamento da FM inclua a redução de peso do paciente obeso.

É evidente que tratamentos dietéticos são uma opção não farmacológica e terapêutica para o controle dos sintomas da FM, e quando bem aplicados, em conjunto com outras formas de tratamento, influenciam nos pacientes com melhoras significativas (RUIZ-CABELLO P, et al., 2017). Cada estilo de dieta tem sua característica e seu ponto forte, e segundo Nadal-Nicolás Y, et al. (2021), Pagliai G, et al. (2020), Lowry E, et 
al. (2020), Ruiz-Cabello P, et al. (2017), Marum AP, et al. (2016) e Batista ED, et al. (2016), apesar dos pontos positivos, as intervenções dietéticas ainda precisam ser aprofundadas e estudadas com afinco a fim de garantir maior segurança das informações a respeito do assunto.

\section{CONSIDERAÇÕES FINAIS}

Hábitos alimentares saudáveis e uma dieta balanceada combinada com exercícios físicos praticados de forma regular são uma forma de tratamento não farmacológico importantepara se obter melhora dos sintomas e, consequentemente, melhora da qualidade de vida dos pacientes com fibromialgia. É evidente que os variados estilos de dietas são formas de terapia para o controle dos sintomas da FM, e quando bem aplicados em conjunto com outras formas de tratamento influenciam nos pacientes com melhoras significativas. Cada estilo de dieta tem sua característica e seu ponto forte, mas apesar dos pontos positivos, ainda precisam ser aprofundadas e estudadas com afinco a fim de garantir maior segurança das informações.

\section{REFERÊNCIAS}

1. ANDRETTA A, et al. Relation between magnesium and calcium and parameters of pain, quality of life and depression in women with fibromyalgia. Advances in Rheumatology, 2019;59(1):1-6.

2. BARKER K. The fibromyalgia story: medical authority and women's worlds of pain. Temple University Press, 2005; $252 \mathrm{p}$.

3. BATISTA ED, et al. Food intake assessment and quality of life in women with fibromyalgia. Revista Brasileira De Reumatologia, 2016;56(2):105-10.

4. BOTTEGA FH, FONTANA RT. A dor como quinto sinal vital: utilização da escala de avaliação por enfermeiros de um hospital geral. Texto \& Contexto Enfermagem, 2010;19 (2): 283-90.

5. BOULTON, T. Nothing and everything: Fibromyalgia as a diagnosis of exclusion and inclusion. Qualitative health research, 2019; 29(6):809-819.

6. CORDERO MD, et al. Clinical sym ptoms in fibromyalgia are associated to overweight and lipid profile. Rheumatology international, 2014;34(3):419-22.

7. CORREA-RODRÍGUEZ M, et al. Dietary inflammatory index scores are associated with pressure pain hypersensitivity in women with fibrom yalgia. Pain Medicine, 2020;21(3):586-94.

8. COSKUN BI. Role of inflammation in the pathogenesis and treatment of fibromyalgia. Rheumatology International, 2019;39(5): 781-91.

9. CRAFT JM, et al. Unique barriers and needs in weight management for obese women with fibromyalgia. Explore, $2015 ; 11(1): 51-8$.

10. HEYMANN RE, et al. New guidelines for the diagnosis of fibromyalgia. Revista Brasileira de Reumatologia, 2017; 57 : 467-76.

11. ISASI C, et al. Fibromyalgia and non-celiac gluten sensitivity: a description with remission of fibromyalgia. Rheumatology international, 2014;34(11):1607-12.

12. JAIME PC, et al. Ações de alimentação e nutrição na atenção básica: a experiência de organização no Governo Brasileiro. Revista de Nutrição, 2011;24(6):809-824.

13. LÓPEZ-RODRÍGUEZ MM, et al. Patterns of food avoidance and eating behavior in women with fibromyalgia. Endocrinología, Diabetes y Nutrición, 2017;64(9): 480-90.

14. LOWRY E, et al. Dietary interventions in the management of fibromyalgia: A systematic review and best-evidence synthesis. Nutrients, 2020; 12(9): 2664.

15. MARTÍNEZ-RODRÍGUEZ A, et al. Efectos de la dieta lacto-vegetariana y ejercicios de estabilización del core sobre la composición corporal y el dolor en mujeres con fibromialgia: ensayo controlado aleatorizado. Nutrición Hospitalaria, 2018;35(2): 392-399.

16. MARTÍNEZ-RODRÍGUEZ A, et al. Psychological and sleep effects of tryptophan and magnesium-enriched mediterranean diet in women with fibromyalgia. International journal of environmental research and public health, 2020;17(7): 2227.

17. MARUM AP, et al.. A low fermentable oligo-di-mono saccharides and polyols (FODMAP) diet reduced pain and improve ddaily life in fibromyalgia patients. Scandinavian journal of pain, 2016;13(1):166-172.

18. MARUM AP, et al. Una dieta baja en oligo-, di-y monosacáridos (FODMAPs) es un tratamiento adecuado para pacientes con fibromialgia, con beneficios clínicos y nutricionales. Nutrición Hospitalaria, 2017;34(3):667-674.

19. NADAL-NICOLÁSY, et al. Vegetarian and Vegan Diet in Fibromyalgia:A Systematic Review. International journal of environmental research and public health, 2021; 18(9): 4955.

20. OLIVEIRA JO, RAMOS JV. Adherence to fibromyalgia treatment: challenges and impact on the quality of life. Brazilian Journal of Pain, 2019;2(1): 81-87.

21. PAGLIAI G, et al. Nutritional interventions in the management of fibromyalgia syndrome. Nutrients, 2020; $12(9): 2525$.

22. PAGLIAI G, et al. Effectiveness of a Khorasan Wheat-Based Replacement on Pain Symptoms and Quality of Life in Patients with Fibromyalgia. Pain Medicine, 2020;21(10):2366-2372. 
23. PAZZI F, et al. Efectos de Ganoderma lucidum y Ceratonia siliqua sobre la glucosa en sangre, el perfil lipídico y la composición corporal en mujeres con fibromialgia. Nutrición Hospitalaria, 2021;38(1):139-145.

24. RODRIGO L, et al. Clinical impact of a gluten-free diet on health-related quality of life in seven fibromyalgia syndrome patients with associated celiac disease. BioMed Central Gastroenterology, 2013;13(1): 1-8.

25. RODRÍGUEZ MD, et al. Patrones de evitación y conductas alimentarias en pacientes con fibromialgia. Endocrinología, Diabetes y Nutrición, 2017; 64(9): 480-490.

26. RUIZ-CABELLO P, et al. Association of dietary habits with psychosocial outcomes in women with fib romyalgia: The al-Ándalus Project. Journal of the Academy of Nutrition and Dietetics, 2017; 117(3): 422-432.

27. SENNA MK, et al. Effect of weight reduction on the quality of life in obese patients with fibromyalgia syndrome: a randomized controlled trial. Clinical rheumatology. 2012;31(11):1591-1597.

28. SIENA LR, MARRONE L. A influência da alimentação na redução ou no agravamento dos sintomas apresentados em pacientes portadores de fibromialgia. Revista Terra \& Cultura: Cadernos de Ensino e Pesquisa, 2018; 25(48-49): 13-9.

29. SILVA AR, et al. Dietary interventions in fibromyalgia: a systematic review. Annals of medicine, 2019; 51(1):2-14.

30. SIRACUSA R, et al. Fibromyalgia: Pathogenesis, Mechanisms, Diagnosis and Treatment Options Update. International Journal of Molecular Sciences, $2021 ; 22(8): 3891$.

31. SLIM M, et al. The effects of gluten-free diet versus hypocaloric diet among patients with fibromyalgia experiencing gluten sensitivity sym ptom s: protocol for a pilot, open-label, randomized clinical trial. Contemporary clinical trials, 2015; 40: $193-198$.

32. SLIM M, et al. The effects of a gluten-free diet versus a hypocaloric diet among patients with fibromyalgia experiencing gluten sensitivity-like sym ptoms. Journal of clinical gastroenterology, 2017;51(6):500-507.

33. SOUZA JB, et al. Prevalence of chronic pain, treatments, perception, and interference on life activities: Brazilian population-based survey. Pain Research and Management, 2017;2017:4643830.

34. VOLTA U. Gluten-free diet in the management of patients with irritable bowel syndrome, fibromyalgia, and lymphocytic enteritis. Arthritis Res Ther, 2014; 16(6): 505. 